\title{
COMPACT SPACES AND PRODUCTS OF FINITE SPACES
}

\author{
DOUGLAS HARRIS
}

\begin{abstract}
It is shown that the compact spaces are precisely the extension closed subspaces of products of finite spaces, where a subspace is extension closed if every open cover of the subspace extends to an open cover of the entire space. Every closed subspace is extension closed, and for Hausdorff spaces the converse also holds. A single compact space $\boldsymbol{U}$ is constructed, such that every compact space is an extension closed subspace of a product of copies of $\boldsymbol{U}$; this parallels precisely the property possessed by the unit interval with respect to compact Hausdorff spaces.
\end{abstract}

Introduction. It is mathematical folklore that compact spaces are finite spaces "made big" in some sense. The following results give exactness to this intuitive feeling. It is shown that the compact spaces are precisely the extension closed subspaces of products of finite spaces. A subspace is extension closed if every open cover of the subspace extends to an open cover of the containing space; this concept includes that of closed subspace, to which it is equivalent for Hausdorff spaces.

In a slightly different direction, a characterization of general compact spaces is given that is an exact parallel to Tychonoff's famous characterization of the compact Hausdorff spaces as the closed subsets of products of the unit interval; a space $\boldsymbol{U}$ is constructed with the property that the compact spaces are precisely the extension closed subspaces of products of $\boldsymbol{U}$.

For simplicity, in the first four sections, the term space will mean a topological space such that distinct points have distinct closures; that is, a $T_{0}$ space. The restriction is not at all essential, and the general case is discussed in $\S 5$.

1. Extension closed subspaces. The subspace $A$ of the space $X$ is extension closed if when $\alpha$ is an open cover of $A$ then there is an open cover $\chi$ of $X$, such that $\alpha=\{V \cap A: V \in \chi$ and $V \cap A \neq \varnothing\}$; that is, every open cover of $A$ extends to an open cover of $X$. Extension closed subspaces, along with a generalization, cluster closed subspaces, are discussed in [2].

Received by the editors November 3, 1971.

AMS 1970 subject classifications. Primary 54D30; Secondary 54C25.

Key words and phrases. Extension closed subspace, universal compact space, degree of completeness.

- American Mathematical Society 1972 
The following four properties of extension closed subspaces are established in [2], and will be used below.

1.1. A closed subspace is extension closed.

1.2. An extension closed subspace of a Hausdorff space is closed.

1.3. An extension closed subspace of a compact space is compact.

1.4. A retract is an extension closed subspace.

An example is given in [2] to show that a compact subspace need not be extension closed.

The following characterization of extension closed subspaces, given in [2], will be used later.

1.5. The subspace $A$ of the space $X$ is extension closed if any filterbase on $A$ that is convergent in $X$ as a filterbase on $X$ is also convergent in $A$.

2. The universal compact space $\boldsymbol{U}$. The ground set of the space $\boldsymbol{U}$ is the collection of sequences $\psi=\left\{\psi_{m}\right\}_{m=1}^{\infty}$ of 0 's and 1's such that for some $j, k \in N, \psi_{j}=0$ and $\psi_{m}=1$ for each $m>k$. Given $k, n \in N$ define $V_{k, n}=$ $\left\{\psi \in \boldsymbol{U}: \psi_{k}=0\right.$ and for some $\left.m>n, \psi_{m}=0\right\}$.

2.1. If $\phi, \psi \in \boldsymbol{U}$ and $\phi \in V_{k, n}$ if and only if $\psi \in V_{k, n}$ for each pair $k, n \in N$, then $\phi=\psi$.

Proof. Suppose $\phi_{k}=0$ and $\psi_{k}=1$. There is $n \in N$ such that, for $m>n, \phi_{m}=\psi_{m}=1$, and it follows that $\phi \in V_{k, n}, \psi \notin V_{k, n}$.

In view of 2.1 the collection $\left\{V_{k, n}: k, n \in N\right\}$ can be defined to be a subbase for the topology of the space $\boldsymbol{U}$.

2.2. Every nonempty open set in $\boldsymbol{U}$ has finite complement.

Proof. It suffices to observe that each $V_{k, n}$ has finite complement.

COROLlaRY. The space $\boldsymbol{U}$ is compact.

Suppose $\alpha=\left\{W_{i}\right\}_{i=1}^{j}$ is a finite open cover of a space $X$. Define $\chi_{\alpha}: X \rightarrow \boldsymbol{U}$ by $\chi_{\alpha}(x)_{m}=0$ if $m \leqq j$ and $x \in W_{m}, \chi_{\alpha}(x)_{m}=1$ if not. Let $\alpha_{U}=\left\{V_{k, j}: k \leqq j\right\}$.

2.3. The function $\chi_{\alpha}$ is continuous, $\alpha_{U}$ is a cover of $U$, and $\chi_{\alpha}{ }^{-}\left(\alpha_{U}\right)=\alpha$.

Proof. It is readily verified that if $k \leqq j$ then $\chi_{\alpha}{ }^{\leftarrow}\left(V_{k, n}\right)=W_{k} \cup$ $\bigcup\left\{W_{i}: n<i \leqq j\right\}$, and if $k>j$ then $\chi_{\alpha}{ }^{-}\left(V_{k, n}\right)=\bigcup\left\{W_{i}: n<i \leqq j\right\}$. The first and last statements follow immediately. To show the second, note that if $\psi \in \boldsymbol{U}$ then $\psi_{m}=0$ for some $m \in N$. If $m>j$ then $\psi \in V_{k, j}$ for each $k \leqq j$, and if $m \leqq j$ then $\psi \in V_{m, j}$.

COROLLARY. Every finite open cover of a space is the inverse image of a finite open cover of a finite space.

Proof. Observe that the subspace $\boldsymbol{U}_{j}=\left\{\psi \in \boldsymbol{U}: \psi_{m}=1\right.$ for $\left.m>j\right\}$ contains the image space $\chi_{\alpha}[X]$.

THEOREM A. A space is compact if and only if it is (homeomorphic to) an extension closed subspace of a product of (spaces homeomorphic to) the space $\boldsymbol{U}$. 
Proof. Let $X$ be any space. Consider the product $\Pi \boldsymbol{U}_{\alpha}$ which consists of copies $\boldsymbol{U}_{\alpha}$ of $\boldsymbol{U}$ indexed by the finite open covers $\alpha$ of $X$. Define $\rho: X \rightarrow \prod U_{\alpha}$ by $\pi_{\alpha} \rho=\chi_{\alpha}$, where $\pi_{\alpha}: \prod \boldsymbol{U}_{\alpha} \rightarrow \boldsymbol{U}_{\alpha}$ is the $\alpha$ th projection; $\rho$ is clearly continuous. To show that $\rho$ is an embedding, it suffices to observe that every open subset $W$ of $X$ is of the form $\rho^{\leftarrow}[V]$ for some open subset $V$ of $\prod U_{\alpha}$; in fact if $\alpha=\{W, X\}=\left\{W_{1}, W_{2}\right\}$, then $V=\pi_{\alpha}{ }^{-}\left[V_{1,2}\right]$ will do. It is clear from 2.3 that the subspace $\rho[X]$ of $\prod \boldsymbol{U}_{\alpha}$ is extension closed, when $X$ is compact. Thus a compact space $X$ is homeomorphic to the extension closed subspace $\rho[X]$ of the product $\Pi \boldsymbol{U}_{\alpha}$ of copies of $\boldsymbol{U}$. The converse assertion is immediate from 1.3 and the Tychonoff product theorem.

THeOREM B. A space is compact if and only if it is (homeomorphic to) an extension closed subspace of a product of finite spaces.

Proof. This follows from the Corollary to 2.3 , using exactly the same method of proof as was used for Theorem A.

3. Degree of completeness. Let $m$ be a finite or infinite cardinal number greater than 1. Let $X$ be a space with cardinal greater than 1. A filter on $X$ is m-cauchy if it contains a member of every open cover of $X$ of cardinal less than $\mathrm{m}$. The space $X$ is $\mathrm{m}$-complete if every $\mathrm{m}$-cauchy filter on $X$ converges, and the degree of completeness of $X$ is the least cardinal $\mathrm{m}$ such that $X$ is m-complete. Also define the degree of completeness of the one point space to be 1 , and the degrez of completeness of the empty space to be 0 .

3.1. A space is compact if and only if it is $\omega_{0}$-complete.

Proof. Suppose the space $X$ is compact; if a filter on $X$ does not converge, it does not contain any member of some open cover of $X$, and therefore it does not contain any member of some finite open cover of $X$.

Conversely, if every $\omega_{0}$-cauchy filter converges, then every ultrafilter converges, and thus $X$ is compact.

3.2. Remark. The preceding proof uses the axiom of choice (in the form of the existence of ultrafilters) to show that an (1) 0 -complete space is compact. As will be seen below, the product of $\omega_{0}$-complete spaces is $\omega_{0}$ complete independently of the axiom of choice, and thus $\omega_{0}$-completeness is a productive property in the absence of the axiom of choice that is equivalent to compactness in the presence of the axiom. For another such property, applying to compact Hausdorff spaces only, see [1].

3.3. An extension closed subspace of an $\mathrm{m}$-complete space is $\mathrm{m}$ complete.

Proof. Suppose $A \subset X$ is extension closed and $X$ is m-complete. If $\lambda$ is an m-cauchy filter on $A$ then $\lambda$ is a base for an m-cauchy filter on $X$. 
Since $X$ is $\mathrm{m}$-complete then $\lambda$ converges in $X$ and thus, by $1.5, \lambda$ converges in $\mathrm{A}$.

3.4. A product is $m$-complete if and only if each of the factors is $m$ complete.

Proof. Suppose $\lambda$ is an $m$-cauchy filter on a product of $m$-complete spaces. Then each projection of $\lambda$ must converge, and therefore $\lambda$ converges. Thus the product is m-complete. The converse follows from 3.3 and 1.4 , since each factor is embeddable as a slice, which is a retract of the product.

It follows from 3.3 and 3.4 that the degree of completeness is an important invariant in connection with universal embedding theorems such as Theorems $\mathrm{A}$ and $\mathrm{B}$. The following results show that spaces exist of any desired degree from 3 to $\omega_{0}$.

Recall from the corollary to 2.3 that $\boldsymbol{U}_{j}=\left\{\psi \in \boldsymbol{U}: \psi_{m}=1\right.$ for $\left.m>j\right\}$.

3.5. For each $j>1$ the space $\boldsymbol{U}_{j}$ has degree of completeness $j+1$.

Proof. For each $k \leqq j$ define $\psi(k) \in \boldsymbol{U}_{j}$ by $\psi(k)_{m}=1$ if $m \neq k, \psi(k)_{k}=0$. It is readily shown that any neighborhood of $\psi(k)$ must contain the neighborhood $W_{k}=V_{k, j} \cap U_{j}$ of $\psi(k)$. Thus $\left\{W_{k}: k \leqq j\right\}$, which is an open cover of $\boldsymbol{U}_{j}$ with $j$ elements, refines every open cover of $\boldsymbol{U}_{j}$. It follows immediately that $\boldsymbol{U}_{j}$ is $j+1$-complete.

For each $k \leqq j$ define $\phi(k)$ by $\phi(k)_{m}=1$ if $m>j$ or $m=k, \phi(k)_{m}=0$ if $m \leqq j$ and $m \neq k$. Let $S=\{\phi(k): k \leqq j\}$. Since $\phi(k) \notin W_{k}$ it follows that $S \not W_{k}$ for any $k \leqq j$. Since $\left\{W_{k}: k \leqq j\right\}$ is a subbase for the topology of $\boldsymbol{U}_{j}$ it follows that the filter $\lambda=\left\{A \subset \boldsymbol{U}_{j}: S \subset A\right\}$ does not converge. However, if $\alpha$ is an open cover of $U_{j}$ with less than $j$ elements it follows from the preceding paragraph that for some $A \in \alpha$ there are distinct $k, i \leqq j$ such that $W_{k} \cup W_{i} \subset A$. Since $S \subset W_{k} \cup W_{i}$ when $k$ and $i$ are distinct, it follows that the filter $\lambda$ is $j$-cauchy.

3.6. The degree of completeness of $\boldsymbol{U}$ is $(1)_{0}$.

Proof. It is straightforward to verify that each $\boldsymbol{U}_{j}$ is a closed subspace of $\boldsymbol{U}$. Combining 1.1, 3.3, and 3.5 it follows that the degree of completeness of $\boldsymbol{U}$ is not finite. Since $\boldsymbol{U}$ is compact then by 3.1 the degree of completeness is $\omega_{0}$.

The degree of completeness for compact Hausdorff spaces is of little interest, as the following result shows.

3.7. The degree of completeness of a compact Hausdorff space with more than one point is 3 .

Proof. Given an open cover $\alpha$ of a compact Hausdorff space $X$, use the normality and the compactness to find a finite open cover $\left\{W_{i}\right\}$ such that for each $W_{i}$ there is $V_{i} \in \alpha$ with cl $W_{i} \subset V_{i}$. Setting $\beta_{i}=\left\{V_{i}, X-\operatorname{cl} W_{i}\right\}$ it follows readily that the meet $\beta$ of the binary open covers $\beta_{i}$ refines $\alpha$. Thus, any 3-cauchy filter contains a member of every open cover, and 
hence converges. Thus every compact Hausdorff space is 3-complete. Further, it is clear that a 2-complete Hausdorff space can have at most one point.

3.8. REMARK. Degree of completeness is a very interesting and useful invariant for compact $T_{1}$ spaces. There exist compact $T_{1}$ spaces of each possible degree. A discussion of such questions for $T_{1}$ spaces is given in [3].

The following simple result will be needed in $\$ 4$.

3.9. The degree of completeness of a space is not greater than its cardinality.

Proof. Clearly every open cover has a subcover of cardinality not greater than the cardinality of the space.

4. Counterexamples. This section consists of remarks and examples to show that Theorems A and B are "best possible" in an appropriate sense.

4.1. Counterexample. For each $n \in N$, the compact space $U$ is not embeddable as an extension closed subspace of any product of finite spaces of cardinal less than $n$.

Proof. Immediate from 3.3, 3.4, 3.9, and 3.6.

REMARK. It follows from 4.1 that in Theorem A, $U$ cannot be replaced by a finite space, and that in Theorem B, no limit can be placed on the cardinality of the spaces employed.

4.2. CountereXAmple. If $V$ is any compact space, there is a compact space $Y$ such that $Y$ is not homeomorphic to any closed subspace of a product of spaces homeomorphic to $V$.

Proof. Choose a regular initial ordinal number $\omega_{\beta}$ greater than the cardinal of $V$; say $\omega_{\beta}=\omega_{\alpha+1}$, where $\omega_{\alpha}$ is the cardinal of $V$. Let $Y_{\beta}$ be the set of ordinals less than $\omega_{\beta}$, and let a neighborhood of any $\delta \in Y_{\beta}$ be any set that contains the tail $T_{\delta}=\left\{\mu \in Y_{\beta}: \mu \geqq \delta\right\}$. Then $Y_{\beta}$ is compact, since the point $0 \in Y_{\beta}$ has the single neighborhood $T_{0}=Y_{\beta}$.

Suppose $\rho: Y_{\beta} \rightarrow \prod V_{k}$ is an embedding of $Y_{\beta}$ into a product of copies $V_{k}$ of $V$. For each index $k$ let $\pi_{k}: \prod V_{k} \rightarrow V_{k}$ be the $k$ th projection, set $\rho_{k}=\pi_{k} \rho$, and let $Z_{k}=\rho_{k}\left[Y_{\beta}\right]$ be the image of $Y_{\beta}$ under $\rho_{k}$. Now if $\delta \leqq \mu \epsilon$ $Y_{\beta}$ then every neighborhood in $Y_{\beta}$ of $\delta$ contains $\mu$, and thus every neighborhood in $Z_{k}$ of $\rho_{k}(\delta)$ contains $\rho_{k}(\mu)$. Now it follows that if $\rho_{k}^{\leftarrow}\left(\rho_{k}(\mu)\right)$ is cofinal in $Y_{\beta}$ then there is $\eta(k)$ such that $T_{\eta(k)} \subset \rho_{k}{ }^{4}\left(\rho_{k}(\mu)\right)$. Since $Y_{\beta}$ is the union of the sets $\left\{\rho_{k}^{\leftarrow}\left(\rho_{k}(\mu)\right): \mu \in T_{\beta}\right\}$, a collection having cardinal less than the regular cardinal $\omega_{\beta}$, some $\rho_{k}{ }^{-}\left(\rho_{k}(\mu)\right)$ is cofinal, and thus $\rho_{k}$ has eventually the constant value $\rho_{k}(\eta(k))$. Also $\rho_{k}(\eta(k))$ is contained in every nonempty open subset of $Z_{k}$. Defining $\eta \in \prod V_{k}$ by $\pi_{k}(\eta)=$ $\rho_{k} \eta(k)$, it follows readily from the properties of the product topology that the trace on $\rho\left[Y_{\beta}\right]$ of the neighborhood filter of $\eta$ is the collection of all nonempty open subsets of $\rho\left[Y_{\beta}\right]$. Thus $\eta \in \operatorname{cl} \rho\left[Y_{\beta}\right]$ and yet $\eta \notin \rho\left[Y_{\beta}\right]$. 
REMARK 1. This counterexample shows that the terms "closed subspace" cannot replace the terms "extersion closed subspace" in Theorem A or Theorem B, no matter what "universal" space is used.

REMARK 2. As is well known any space $X$ is embeddable as a subspace of a product of copies of the two point connected space (Sierpiński space). According to [2] the embedding is as a cluster-closed subspace if and only if the space $X$ is compact. This gives an embedding theorem and characterization of compactness in terms of Sierpiński space, but the embeddings usually lack desirable properties; for eximple such an embedding is extension closed if and only if the space has degree of completeness 2 or less.

5. General spaces. The restriction to what are usually called $T_{0}$ spaces was made for purposes of simplicity only, and may readily be removed at the cost of some complication in the statement of results. The universal space $\boldsymbol{U}$ may be replaced with a compact space containing $\boldsymbol{U}$ as an extension closed subspace and containing a ropy of the two point trivial (=indiscrete) space $\boldsymbol{T}$. The topological union of $\boldsymbol{U}$ with $\boldsymbol{T}$, or the topological product of $\boldsymbol{U}$ and $\boldsymbol{T}$, will do nicely. The copy of $\boldsymbol{T}$ is used to obtain a one-one map when embedding, and the copy of $U$ is used to obtain the extension closed property of the embedding. All of the preceding results are readily adapted to the general case.

\section{REFERENCES}

1. W. W. Comfort, A theorem of Stone-Čech type, and a theorem of Tychonoff type, without the axiom of choice; and their real compact an'logues, Fund. Math. 63 (1968), 97-110. MR 38 \#5174.

2. D. Harris, Extension closed and cluster closed subspaces, Canad. J. Math. (to appear).

3. - Universal compact $T_{1}$ spaces, General Topology and Appl. (to appear).

Department of Mathematics, Marquette University, Milwaukee, Wisconsin 53233 